\title{
Highlights from the Faraday Discussion on New Directions in Porous Crystalline Materials, Edinburgh, UK, June 2017
}

\author{
Matthew A. Addicoat* \\ School of Science and Technology, Nottingham Trent University, Clifton Lane NG11 8NS \\ Nottingham, UK \\ e-mail: matthew.addicoat@ntu.ac.uk
}

Thomas D. Bennett*

Department of Materials Science and Metallurgy, University of Cambridge, Cambridge CB3

OFS, UK

e-mail: tdb35@cam.ac.uk

\section{Ivo Stassen*}

Centre for Surface Chemistry and Catalysis, KU Leuven - University of Leuven,

Celestijnenlaan 200F 3001 Leuven, Belgium

e-mail: ivo.stassen@kuleuven.be

A lively discussion on new directions in porous crystalline materials took place in June 2017, with the beautiful city of Edinburgh as a backdrop, in the context of the unique Faraday Discussions format. Here, 5 minute presentations were given on papers which have been submitted in advance of the conference, with copious time allocated for in-depth discussion of the work presented. Professor Mircea Dincă (MIT), chair of the scientific committee, opened the conference by welcoming the many different nationalities attending, and outlining the format of discussions.

\section{Opening Lecture}

Professor Omar Yaghi (UC Berkeley) was invited to give the opening lecture, in which he sought to put metal-organic frameworks (MOFs) in context with both zeolites and organic complexes, alongside important early work on hybrid coordination polymers by Kinoshita and Kitagawa. The colour choice of the ubiquitous 'yellow' sphere to illustrate the porosity within MOFs was clarified, and linked to the sunny Arizonan weather. Early industrial interest in the MOF field was also highlighted, with a quote from Ulrich Mueller from BASF SE "The synthesis of MOF-5 broke the surface area record for porous carbon, and the number was so unbelievably high, I thought it had to be a misprint".

The chemical variety of MOFs in the literature was demonstrated by a discussion of HKUST1, UiO-66, MILs, tetrazolates, pyrazolates and catecholates, amongst others. A highly humorous historical see-saw of the porosity record for ultra-high porous MOFs ended with congratulations for Professor Omar Farha (Northwestern University), who it was generally agreed holds the record for most porous MOF (NU-110) at present. Such high porosities lend themselves will to applications in gas sorption, separation and catalysis. In particular, the promise of MOFs in solving global challenges, such as providing clean drinking water to the one third of the population without it at present, was emphasized. Whilst many MOFs display high water adsorption capacities, MOF-801 combines this with the ability to subsequently release it again under mild conditions. Current progress is exemplified by the $2.8 \mathrm{~L}$ of water delivered per Kg of MOF per day, though the task of 'seeing things through the eye of the molecule' has only really just begun. Further applications of reticular materials in catalysis and photocatalytic conversion, made possible by MOFs occupying the space between beautiful 
heterogeneous catalysts which are robust but not designable, and biological enzymes which are exquisitely designed but not robust, are also promising.

To enable this, increasing complexities within MOFs, and 'diversity within order' is often sought, through the creation of e.g. defects and solid solutions. Such strategies do however lead to a large range of diverse phenomena such as breathing, gate-opening and negative adsorption under pressure. For this reason, the reticular synthesis of covalent organic frameworks (COFs) from design principles is perhaps easier than that of their hybrid counterparts.

\section{Session 1: Electronic, magnetic, and photophysical properties of MOFs and COFs}

The first session, on the electronic, magnetic and photophysical properties of MOFs and COFs, was chaired by enigmatic Chris Hendon (University of Oregon), and started by Professor Jing Li (Rutgers University) with a presentation on functionalizing MOFs for the capture of radioactive organic iodides (DOI: 10.1039/c7fd00013h). An interesting strategy of enhancing the uptake capacity of MIL-101 (Cr) for $\mathrm{CH}_{3} \mathrm{I}$ capture by grafting amines onto frameworks was presented. Three amine molecules, N,N-dimethylethylenediamine (DMEDA), N,N-dimethyl1,3-propanediamine (DMPDA), and $\mathrm{N}, \mathrm{N}$-dimethyl-1,4-butanediamine (DMBDA) were selected. Through direct addition into the synthesis of MIL-101, frameworks in which the amines were connected directly to the metal SBU produced. The results revealed that DMEDA functionalized MIL-101-Cr gave rise to a record-high CH3I saturation uptake capacity of $80 \%$ at $150 \mathrm{C}$, due to increased chemi- and physisorption.

The stability of such materials, and not just the performance, is of course important. The synthesis of an ultra-stable metal-organic framework with excellent acid and base resistance was presented by Professor Guangshan Zhu (Northeast Normal University) (DOI: 10.1039/c7fd00017k). The framework of composition $\left[\mathrm{Cu}_{2}(\mathrm{DCI})_{2}\right](\mathrm{MeCN})$ (DCI dicyanoimidazolate) prompted a lively end of session discussion, which focused much on the chemical and thermal stability of MOFs. In particular, the role that X-ray powder diffraction plays in determining the chemical stability of a structure was scrutinized. Real care should be taken to identify subtle polymorphism or defect creation after chemical soaking, and the possibility of partial dissolution of the MOF after chemical soaking (which would not be identified by PXRD), was further emphasized. Secondly, the role of TGA in determining thermal stability was raised - such measurements do not identify phase transitions which proceed without mass loss, and should be combined with differential scanning calorimetry (DSC) or variable temperature X-ray measurements.

The first afternoon session was concluded by Professor Monique van der Veen (Delft University of Technology), who looked at water splitting by $\mathrm{NH}_{2}-\mathrm{MIL}-125$, which is the best performing $\mathrm{d}^{0}$ transition metal MOF (DOI: 10.1039/c7fd00029d). Several amine-type species were physisorbed into the structure (Figure 1), and proven to act as charge acceptors after visible light illumination. 


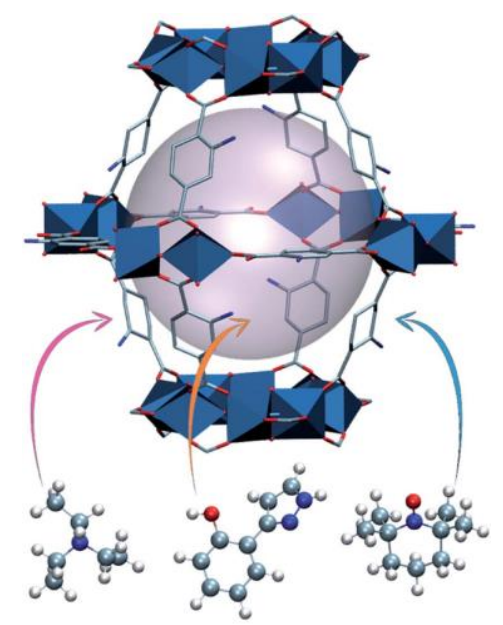

Figure 1. Schematic representation of charge carrier molecules occluded into the $\mathrm{NH}_{2}-\mathrm{MIL}-$ 125 structure. Reproduced from DOI: 10.1039/c7fd00029d with permission from the Royal Society of Chemistry.

\section{Session 2: New direction in gas sorption and separation with MOFs}

The second afternoon session, expertly chaired by Professor Nathaniel Rosi (University of Pittsburgh), concentrated on the more traditional areas of gas sorption and separation. Dirk Volkmer (Augsburg University) opened by describing a cyclic gas-phase heterogeneous process in a metal-organic framework involving a nickel nitrosyl complex (DOI: 10.1039/c7fd00034k). Modification of MFU-4l ([ $\left.\mathrm{Zn}_{5} \mathrm{Cl}_{4}(\mathrm{BTDD})_{3}\right], \mathrm{H}_{2}$-BTDD = bis $(1 \mathrm{H}-$ 1,2,3-triazolo[4,5- $b],\left[4^{\prime}, 5^{\prime}-i\right]$ )dibenzo[1,4]dioxin) was performed and terminating chloride ligands exchanged for $\mathrm{NiNO}_{2}$ functionalities. The resultant Ni-MFU-4l-NO 2 framework undergoes reduction by $\mathrm{CO}$, to Ni-MFU-41-NO. This latter framework then reacts with NO to return to the Ni-MFU-41- $\mathrm{NO}_{2}$ state. This cyclic process, $2 \mathrm{NO}+\mathrm{CO}=\mathrm{N}_{2} \mathrm{O}+\mathrm{CO}_{2}$, proceeds with retention of catalyst crystallinity, though activity reduced to $50 \%$ of its original value after 10 cycles due to the build-up of contaminants within the catalyst.

Given the pervasive debate on the industrial applicability of MOFs, the last talk of the day, by Dr. Sheena Hindocha of Johnson Matthey, offered an engaging discussion on the study of the scale-up, formulation, ageing and ammonia adsorption capacity of MIL-100(Fe), Cu-BTC and CPO-27(Ni) for use in respiratory protection filters (DOI: 10.1039/c7fd00090a). The physical shaping of MOF structures is increasing in prominence in the literature due the implications of different morphologies for industrial application. Two strategies to shape the three MOFs were presented, including palletization by compaction of the pure samples, and also mixing the pure samples with a binder prior to compaction. $\mathrm{Cu}-\mathrm{BTC}$ was observed to be the least resistance to compaction, with degradation under repeated compaction, or compaction with a binder. This resulted in a decreased capacity of $37 \mathrm{mg} \mathrm{g}^{-1}$ from $97 \mathrm{mg} \mathrm{g}^{-1}$ pre-forming. MIL-100(Fe) and $\mathrm{CPO}-27(\mathrm{Ni})$ retained crystallinity upon the forming process, and displayed ammonia adsorption capacities of $47 \mathrm{mg} \mathrm{g}^{-1}$ and $62 \mathrm{mg} \mathrm{g}^{-1}$ respectively. Ageing was also studied at 25 ${ }^{\circ} \mathrm{C}$ and $80 \%$ humidity for one week, resulting in a decrease in capacities to $36 \mathrm{mg} \mathrm{g}^{-1}$ and 60 $\mathrm{mg} \mathrm{g}^{-1}$ respectively.

\section{$\underline{\text { Session } 2 \text { (continued) }}$}


Session 2 continued the next morning, with Professor Omar Farha taking over the chair. The session largely ignored the stated theme of gas sorption and separation. Professor Mohamed Eddaoudi (KAUST) began the day by describing 'on-purpose' MOFs (DOI: 10.1039/c7fd00119c). In an 'on-purpose' MOF, a target highly-connected net is identified and particular care is taken to synthesise complex building blocks that in turn occupy the highlyconnected sites in the desired net. In particular, he presented the synthetic strategy that yielded a 12-connected double six-membered ring (d6R) unit, which when combined with a square (4connected) building block, forms the $(4,12)$-coordinated shp net and when combined with a trigonal prism (6-connected) building unit, forms the $(6,12)$-coordinated alb net (Figure 2). These two nets are the only known two edge-transitive nets with a vertex figure as a d6R. Finally, he enumerated several different ways of building up the d6R, square and trigonal prism units themselves, and the derived nets that would be achieved in each case. At the end of his presentation, he challenged the audience, excluding his co-authors, to occupy the entire 20 minutes allocated to questioning, a challenge the audience happily accepted and met.

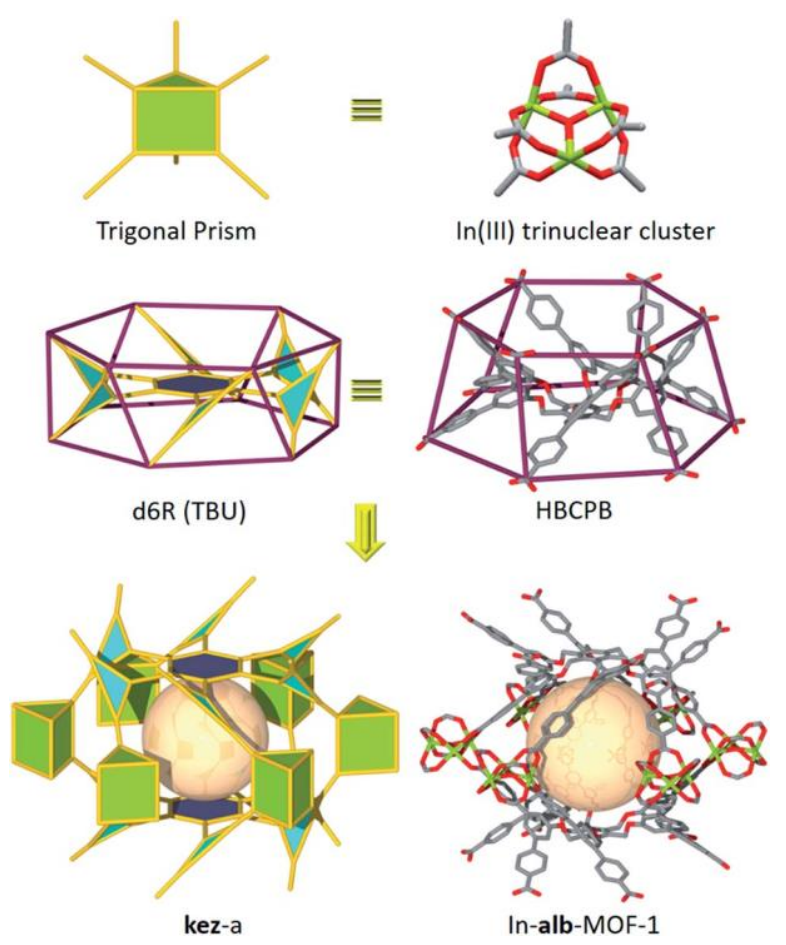

Figure 2. MOFs based on trigonal prism (6-connected) and d6R building units. Reproduced from DOI: 10.1039/c7fd00119c with permission from the Royal Society of Chemistry.

A lively discussion followed in which "all MOFs look the same" vs. "Not all MOFs are equal" was debated. Prof. Eddaoudi posited that functionality was the future because functionality over any other property defines what each MOF could actually do. The historical trend for MOFs to be discovered rather than designed was noted, but Prof. Eddaoudi felt the two were linked, particularly for very highly connected nets. In his work, determining the synthetic conditions to build a high-connected node was half the battle, and that once this node was synthesized, building the unique MOF with that building block was comparatively straightforward, therefore this was indeed a design process that is truly unique to MOF chemistry.

Returning to the session theme, Dr. Karen Leus (Ghent University) described the performance of UiO-66-(SH) $)_{2}$ for $\mathrm{Hg}$ removal from water in environmental conditions (DOI: 10.1039/c7fd00012). The 2,5-dimercaptoterephthalic acid $\left(\mathrm{H}_{2} \mathrm{BDC}-2,5 \mathrm{SH}\right)$ linker was 
synthesized and then used in place of BDC in the literature synthesis of UiO-66. UiO-66- $(\mathrm{SH})_{2}$ was observed to adsorb $236.4 \mathrm{mg} \mathrm{g}^{-1}$ of $\mathrm{Hg}(\mathrm{II})$, a figure over 8 times that of the pristine UiO$66\left(28.7 \mathrm{mg} \mathrm{g}^{-1}\right)$ and functioned over a broad $\mathrm{pH}$ range of $3-11$, even in the presence of potentially interfering $\mathrm{Cu}$ and $\mathrm{Fe}$ ions. The framework also cycled well, with only a slight reduction in desorption efficiency after the third cycle. Discussion focused on the role of the thio groups and the extent of decomposition in the structure.

The final speaker of the session, Professor Nathaniel Rosi asked (and answered!) how MOF structures could be built in order to control the direction of molecular transport (DOI: 10.1039/c7fd00045f). 'bMOF-100' consists of Zn-tetrahedral units, each linked by three, 1,1'biphenyl-4,4'-dicarboxylic acid ( $\mathrm{H}_{2}$-BPDC) linkers, a unique feature which facilitates linker exchange without the framework disintegrating. Interestingly, there is some degree of flexibility in the size of linkers that can be replaced, allowing sequential crystal to crystal reactions: Firstly the BPDC linker in bMOF-100 could be exchanged for azobenzene-4,4'dicarboxylic acid $\left(\mathrm{H}_{2}-\mathrm{ABDC}\right)$ resulting in bMOF-102. The question was then posed as to how this transformation occurs. Time dependent PXRD indicated that in 10 minutes, $38.7 \%$ of the linkers were exchanged. Breaking a large crystal after the exchange reaction showed distinct onion-like domains, supporting the hypothesis that linkers were gradually exchanged from the outside in. With this knowledge of a two-layer onion, he then presented their work on a ternary gradient bMOF, where ABDC linkers could be replaced with the even longer 2'-nitro1,1':4',1' '-terphenyl-4,4' '-dicarboxylic acid $\left(\mathrm{H}_{2}-\mathrm{NO}_{2}\right.$-TPDC) yielding a domain of bMOF106. In conclusion, the linker size and flexibility was key to attaining the desired superstructure - flexible and same size linkers could mix freely, a sufficient size difference in linkers leads to core-shell structures which could then result in one-way transport of guest molecules. In the discussion, the kinetics and mechanism of linker exchange in the MOF was extensively discussed (e.g. relying on existing defects, local dissolution and reformation, straight exchange). Participants lamented the limits of the existing spectroscopy and several possible experiments to further elucidate the gradient structure were put forward.

\section{Session 3: MOFs modeling and theory}

After participants were re-caffeinated, the third session, chaired by Professor Aron Walsh (Imperial College London) began, themed "MOFs Modelling and Theory". Professor Laura Gagliardi (University of Minnesota) presented a very challenging system of NU-1000 decorated by $\mathrm{Al}$ and then used to support Ir catalysts for ethylene hydrogenation (DOI: 10.1039/c7fd00031f). Challenges included modelling the $v(\mathrm{CO})$ on the Ir atoms within the pore (as the best descriptor of the cluster), which despite careful modelling with many functionals and basis sets currently did not appear to correlate with the catalytic activity. Likewise, the calculated partial charges on the Ir atoms failed to correlate with catalytic activity. She also presented a mechanistic pathway for hydrogenation of ethylene to ethane by a $\operatorname{Ir}(\mathrm{C} 2 \mathrm{H} 4) 2(\mathrm{H} 2)$ catalyst immobilised on NU-1000 and Al-decorated NU-1000, but these showed a very high barrier, which seemed to suggest the reaction would not be possible. She invited discussion on these not-so-conclusive conclusions.

Dr. Keith Butler (University of Bath) moved on to predictive computational design of MOFs and in particular, MOF thin film devices (DOI: 10.1039/c7fd00019g). When considering a device, "the interface is the device", therefore, designing the interface is paramount. The screening procedure identified the growth planes of each material and then considered the electronic structure (carrier transport), chemical match (bonding), lattice match and finally the interface contact resistance (band alignment, Figure 3). After consideration of all these factors, 
the combinations of ZIF-8 on Zn and ZIF-67 on Co were highlighted as most suitable for further pursuit.

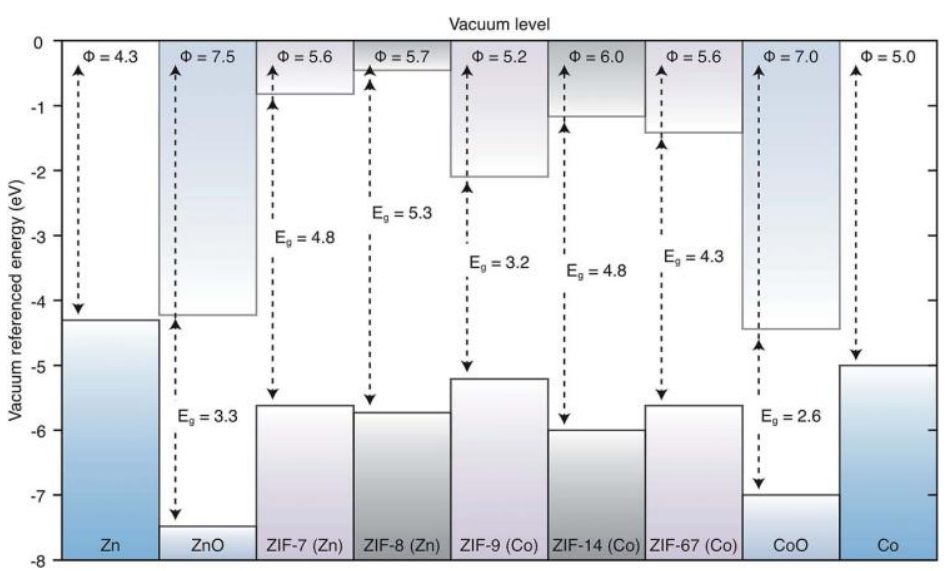

Figure 3. First-principles quantum mechanical electronic band alignment calculations for ZIFs and respective oxides. The work functions of the contact metals are given at either end. Reproduced from DOI: 10.1039/c7fd00019g with permission from the Royal Society of Chemistry.

Professor Chris Wilmer (University of Pittsburgh) flew in just in time to end the session by discussing what we might learn from the structure-property relationships in pseudomaterials (DOI: 10.1039/c7fd00038c). Beginning from the starting point of the hypMOF database, containing 130,000 hypothetical MOFs, the following five questions were posed:

1. Which are the best MOFs?

2. What are the structure-property relationships?

3. Are the boundaries due to physics or merely limited data?

4. What accounts for shape and location of boundaries?

5. Are the limits universal to porous materials or just MOFs?

The question is then how to determine the theoretical limits for the properties of porous materials. The best MOF represents a lower bound to this limit. Rather than try to enumerate all possible MOFs, a more encompassing approach to understand these limits is to consider pseudomaterials composed of randomly arranged Lennard-Jones spheres in periodic cells.

An engaging discussion of the three papers followed in which the interplay between experiment and modelling was highlighted, that experiments (e.g. EXAFS) could narrow the possibilities for modelling and modelling could often provide detail inaccessible to experiment. The representativeness and correctness of models was debated, particularly noting that models are typically minimised to a local minimum, but this may not be the correct minimum and equally, the global minimum structure may not be one actually present in the experiment. Often further modelling or experiments were needed to resolve the dispute. An example from Gagliardi's paper was highlighted, in that experiment shows the number of $\mathrm{Al}$ atoms per node, but not their distribution - while acknowledging the importance of the distribution, it was noted to be difficult to model, simply because of the vast phase-space. It was also noted that the modelling replicated trends very well, but struggled with the exact numbers and that perhaps increasing the size of the model or employing a periodic model would improve the agreement.

The difficulty in replicating exact numbers rather than trends continued when discussing the next paper. The band alignment of the base materials themselves could be calculated accurately, but the band alignment in the device depends sensitively on the interface itself. 
Deformation potentials can be quite large and dipoles forming at the interface allows charge transfer and can shift the offsets. It was pointed out that for a screening application, this was considering the materials independently was about the best one could do as calculation of the interface was expensive and furthermore there was a combinatorial explosion inherent in considering all possible interfaces.

Dr. Chris Hendon (whilst being an author on the paper) criticised the consideration of strain, when the metal had such a small cell size and could readily be extended to near-match the MOF cell size and equally, the porous material is sufficiently flexible, thereby able to accommodate a lot of strain. The discussion then turned to an essential difficulty of characterisation vs modelling by asking what if an oxide layer was actually responsible? In that case, the highquality modelling presented would still be modelling the wrong thing. Finally, the need for global optimisation of the interface was highlighted.

Concluding the discussion of the session papers, the capacity for chemistry in the pseudomaterial approach was questioned. Dr. Wilmer pointed out in response, that the scope of the study was more about the underlying physics and determination of the absolute limits of porous materials. Furthermore, while there certainly was potential to extend the model to incorporate chemisorption, it would be at the cost of making the model unreasonably expensive and would narrow the scope of the insights gained. Finally Dr. Wilmer pointed out that the shapes of the structure-property relationships were the same for the current pseudomaterials as for the hypMOFs - thereby supporting the fact that pseudomaterials are actually yielding useful insight as a general superset of porous materials (including MOFs).

A very lively general discussion followed: Prof. Omar Yaghi postulated that perhaps the primary aim of computational chemistry should be to directly guide the synthesis of predicted structures through reagent suggestion, and that there was much more room for computational colleagues to direct the approach of synthetic researchers, rather than confirm or rationalize. Prof. Laura Gagliardi responded by highlighting the need to validate models, and showed an example of a predicted new MOF, which had then been synthesized. Prof. Mircea Dincă added that the role of chemical intuition may get us so far in the synthesis of new structures, but predicting and directing the synthesis of structures outside of this intuition-space was more of a challenge. The need for modelling at different levels to narrow down the phase space was highlighted. Prof. Aron Walsh suggested that there is a need to re-evaluate what we model that useful models need to include thermal motion and defects. Prof. Gagliardi extended this point, noting the lack of precision in even "atomically precise" structures - and the great challenge this presents in using the experiment as a starting point for modelling. It was agreed that fluxionality and combinatorial explosions present in MOFs is an opportunity for both experiment and modelling.

To bring together the two areas, it was suggested that we need to redefine what useful information from theory and calculation actually is, and work around tractable questions. It is unlikely that, without some parameter space, theory is capable of nominating a particular MOF material and providing a descriptive methodology of realizing it. The importance of remembering that every model is based on assumptions, to both experimental and computational colleagues, was stressed. Dr. Miguel Jorge (University of Strathclyde) affirmed that whilst historically, comparisons between the available surface area, diameter, cell volume and length have been important, it is important for us to remember that not all structures are perfect, some are defective, and that they all have a role to play. In order to let everyone go to lunch, the discussion was concluded by highlighting the essential role of theory in explaining, 
interpreting and supporting experimental results. The interaction between experimental and computational researchers needs to be truly interactive in both directions.

\section{Session 4: Catalysis in MOFs}

After lunch, the fourth session began around the theme of "Catalysis in MOFs", chaired by Prof. Dincă. Dr. Frederick Haase (Max Planck Institute for Solid State Research, LudwigMaximilian University of Munich) described artificial photosynthesis for solar fuel production (DOI: 10.1039/c7fd00051k). COFs are an ideal template for soft photocatalysts due to their flexibility - in particular the ability to functionalise at will. Previously, a series of aryl triphenyl azine COFs (N-x COFs) showed a notable increase of $\mathrm{H}_{2}$ evolution with increasing nitrogen content in the COF. In the new COF presented, PTP-COF, the nitrogen atoms now reside on the outside of the SBU. The rate of $\mathrm{H}_{2}$ evolution from this reverse polarity COF was found to be approximately the same as N1-COF.

Prof. Carlo Lamberti (University of Turin) presented a synergistic combination of experiment and theory (DOI: 10.1039/c7fd00024c). By switching the biphenyl linkers in UiO-67 with bipyridine (bipy), $\mathrm{PtCl}_{2}$ could be added to up to $10 \%$ of linkers which was proven by both EXAFS and XANES spectroscopy. The embedded Pt atoms were remarkably reactive; reaction with toluene-3,4-dithiol (H2tdt) was shown to exchange the two $\mathrm{Cl}$ ligands with two sulfur atoms from the $\mathrm{H}_{2}$ tdt and liquid-phase oxidative addition of $\mathrm{Br}_{2}$ resulted in $\mathrm{Pt}(\mathrm{IV})$ (Figure 4). For both reactions the modelled XANES spectra agreed very well with the experimental spectra and confirmed the interpretation of the results. Copper atoms could be added to the bipy linkers in the same manner, but in contrast to $\mathrm{PtCl}_{2}$, each embedded $\mathrm{Cu}$ (II) atom possessed one $\mathrm{Cl}$ and one $\mathrm{OH}$ ligand.

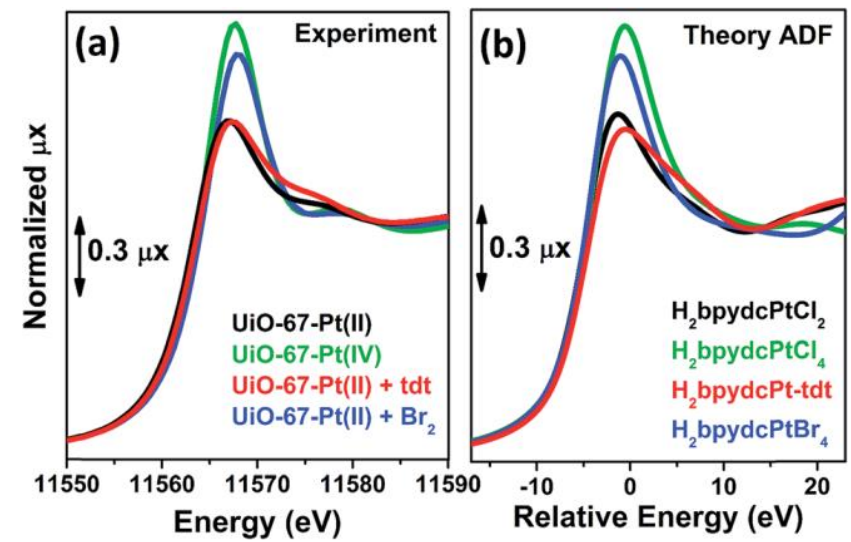

Figure 4. (a) Experimental and (b) modelled XANES spectra of bipy UiO-67 loaded with $\mathrm{PtCl}_{2}$ and $\mathrm{PtCl}_{4}$. before and after interaction with $\mathrm{H}_{2}$ tdt and $\mathrm{Br}_{2}$. Reproduced from DOI: $10.1039 / \mathrm{c} 7 \mathrm{fd} 00024 \mathrm{c}$ with permission from the Royal Society of Chemistry.

Finishing the session, Connie Lu (University of Minnesota) presented intriguing work aimed at achieving a strong and stable uniform 3D-grid of catalytic sites (DOI: 10.1039/c7fd00055c). NU-1000 was the ideal substrate for such an endeavour because of its large (3nm wide) channels and $\mathrm{Zr}$ nodes which offer many $-\mathrm{OH}$ groups which may anchor bimetallic clusters. Onto this well-known MOF, (py 3 tren)AlCoMe and (py3tren) $\mathrm{Co}_{2} \mathrm{Me}$ were anchored. Recognising that a weakness of using MOFs as a support for catalysis is that they often degrade under the desired reaction conditions, the NU-1000 MOF was then cast with silica and calcined, leaving the NU-1000 Zr6 oxide clusters and the Co/Al catalytic clusters remain accessible and active in a robust silica support. 
In general discussion, Prof. Dinca asked what we should do with MOFs in order to compete with heterogeneous catalysts. Dr. Marco Ranocchiari (Paul Scherrer Institute) suggested that MOFs were already competitive, in particular, we should look for interesting cases such as reversing entioselevtivity. Prof. Dinca responded that while several proof-of-concepts existed, what should we do to commercialise them? Prof. Wenbin Lin (University of Chicago) noted that his strategy has evolved over the last decade and suggested to look at commodity chemicals that were produced on the 10-20 tonne scale, but steer away from petrochemicals as they were too large scale. Prof. Omar Farha questioned this, asking why we shouldn't aim our sights at such large-scale reactions - there was nothing in principle stopping MOF chemistry from presenting a better catalyst, active at a lower temperature than some existing catalyst. Prof. Lamberti, sagely reminded everyone that many zeolite catalysts had taken more than 20 years to come to market. Any new catalyst would probably require new plants and these require time and investment. His suggestion was to look toward the pharmaceutical industry as a place where MOFs could easily contribute as the desired reactions were comparatively small-scale and high value. It was pointed out that MOFs could create unusual species such as almost naked Pd atoms that are not possible in solution or heterogeneous catalysis and these unusual and unique species should be employed for otherwise impossible reactions.

\section{Session 4 (continued)}

After an reenergizing tea break, the session on catalysis was continued by Prof. Wenbin Lin with a presentation on phenantroline (PT) based MOFs for Fe-catalyzed $\mathrm{C}_{\mathrm{sp}}{ }^{3}$-amination (DOI:

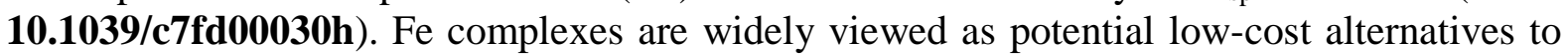
replace precious metals. The catalytically active Fe(II) sites in the PT-MOFs were constructed by postsynthetic metalation of the linkers with $\mathrm{FeBr}_{2}$ (Figure 5). Catalytic activity was demonstrated in amination of secondary allylic and benzylic $\mathrm{C}-\mathrm{H}$ bonds and after reduction of the active site in intramolecular amination of aliphatic azides.
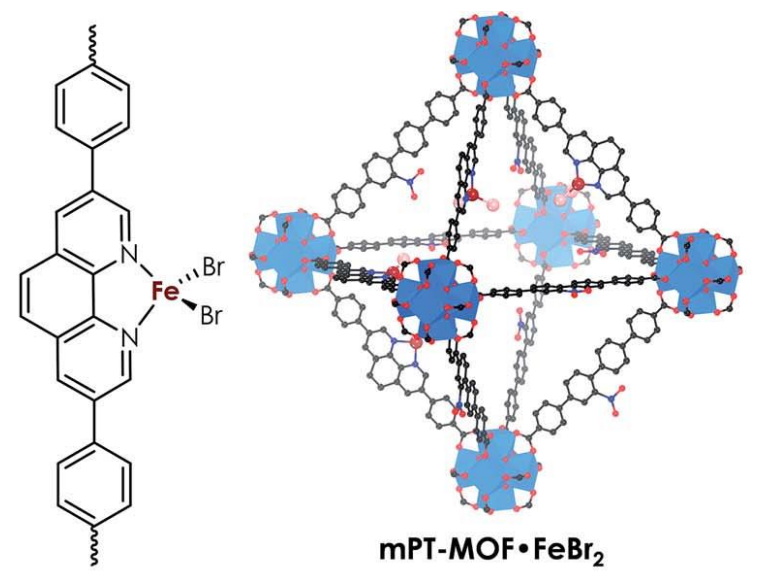

Figure 5. Representation of catalytically active Fe(II) site and PT-MOF structure. Reproduced from DOI: 10.1039/c7fd00030h with permission from the Royal Society of Chemistry.

After an initial discussion about the oxidation states of Fe during the reaction mechanism and about the 'dream substrate scope' of amination catalysts, the conversation moved on to a broad debate about the various aspects of MOF catalysis on the molecular scale. A question asked from the audience, directed to the 'older' - experienced — academics, instigated an existentialist exchange of ideas: should catalytic research in academia be aimed at curiositydriven exploration and deep insight or should it focus mainly on industrial needs? Working 
towards industrial products is important to justify the branch of research to the society, however fundamental research should not be overlooked in an academic setting. One of the concluding statements by the chair stressed the importance of honest communication of motivation (particularly when writing the introduction section of articles).

The session was concluded by Prof. Shengqian Ma (University of South Florida) who presented work on Pd nanoparticles immobilized in MIL-101 MOFs which showed different degrees of hydrophilicity through $\mathrm{SO}_{3}$ functionalization of the linker (DOI: 10.1039/c7fd00015d). Using the hydrophilic catalyst supports, improved kinetics were achieved in hydrodeoxygenation of vanillin in water. This effect was linked to the favorable adsorption of hydrophilic oxygen-rich reagents and desorption of more hydrophobic hydrodeoxygenated products. Food for thought during the discussion were the intricacies of water-phase heterogeneous catalysis and upgrading of reagents derived from rather insoluble biomass (vanillin being a common component in lignin-derived biofuel), and the suitable pore size range of MOFs in this context.

\section{Conference dinner}

The dinner was preceded by the second poster session of the conference, with poster prize winners Zhijie Chen (KAUST) and Laura Ascherl (Ludwig-Maximilian University of Munich) congratulated. The conference dinner was held at the atmospheric Ghillie Dhu auditorium at the West End of Edinburgh's City Centre. The dinner was attended by the president of the Faraday Division, Eleanor Campbell. At this occasion, Prof. Omar Yaghi was presented with the Spiers Memorial Award 2017 for 'pioneering the conceptual and experimental basis of crystalline metal-organic frameworks and covalent organic frameworks with exceptional porosity and applications'. Prof. Laura Gagliardi was presented with the Bourke Award 2016 for 'contributions to quantum chemistry aimed at predicting new materials for catalysis, gas separation and at describing excited state phenomena'. As a last chapter of the evening, the president started the famous Faraday Discussion Loving Cup ceremony. The cheerfulness of this traditional activity was appreciatively carried on by the dinner attendants.

\section{Pictures from conference organizers}

Figure 6. Conference dinner/prizes

\section{Session 4 (concluding)}

A third session about catalysis in MOFs was chaired by Professor David Harris (Northwestern University) on the final morning of the conference. The session was started by Prof. Matt Rosseinsky (University of Liverpool) with a presentation on peptide-inspired structurally flexible In-MOFs using $\mathrm{sp}^{3}$-containing amide-functionalized linkers (DOI: 10.1039/c7fd00085e). The rotation of the carbon single bonds provides a strategy towards construction of enzyme-inspired complex energy landscapes. The characterization challenges in research of these structurally flexible systems were delineated by the panel and the audience in the following discussion. Indeed, highly dynamic behavior can complicate the interpretation of cornerstone methods such as powder X-ray diffraction and gas physisorption. Moreover, topology-driven approaches to structure prediction are typically not working in this area, and alternatives methods will need to be developed.

The second speaker of the session was Dr. Karen Chapman (Argonne National Lab), who presented work on real-time $\mathrm{X}$-ray diffraction characterization of $\mathrm{Cu}-\mathrm{O}$ active site formed during functionalization of NU-1000 using atomic-layer deposition (DOI: 10.1039/c7fd00110). The work showcased complementary use of 'big picture scale' Bragg diffraction and 'atomic resolution' pair distribution function (PDF) structure refinement 
techniques, to distinguish formation of $\mathrm{Cu}-\mathrm{O}$ nanoparticles of two distinct sizes (Figure 7). The discussion ranged from practical aspects such as preventing X-ray damaging during realtime experiments, to the general mechanisms of ion mobility within MOFs.

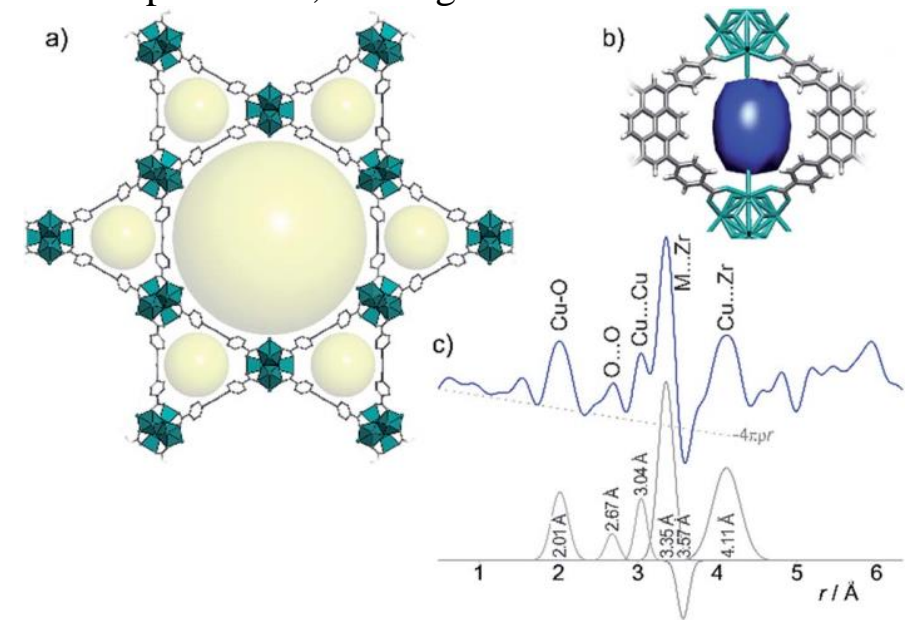

Figure 7. Representation of (a) the NU-1000 structure (viewed along the c-axis). (b) X-ray diffraction density map showing location of $\mathrm{Cu}-\mathrm{O}$ nanoparticles in the small pores. (c) Differential PDF showing atom-atom distances in the $\mathrm{Cu}-\mathrm{O}$ loaded MOF.

The session was closed by Professor Jeff Long (UC Berkeley), who presented work on Ni(II) 2,2-bypyridine catalytic sites immobilized in UiO-66 type materials (DOI: 10.1039/c7fd00061h). Analysis of the performance of these catalysts in ethylene oligomerization revealed non Flory-Schulz contributions to the turnover frequency. The observed tuning of the balance between propagation and termination reactions is related to pore confinement. However, the mechanism proved more complex than initially anticipated, as active sites of multiple natures were observed. Eventually, the discussion shifted to the work on magnetism of the speaker. Statements about 'the dream of building qubits into organized MOF lattices' completed a figurative cycle, as the debate unexpectedly arrived once again at the formal topic of the first session.

\section{Concluding remarks lecture}

During the final session of the conference, Prof. Kitagawa (Kyoto University) gave an invited lecture about the present state of affair and the future of 'the chemistry of the nanospace'. The significance of designability and diversity of crystalline porous materials was highlighted: organic and inorganic building blocks can be tuned to assimilate properties typical to soft either hard materials. Next, the evolution of third generation porous crystals from second and first generation compounds was historically revisited. At the start of the $21^{\text {st }}$ century, second generation porous coordination polymers enabled design strategies for topology, surface and permanent porosity. Subsequently, third generation 'soft porous crystals' provided access to some of the most exciting properties that have become the cornerstone many applications envisioned by the community: broad chemical diversity, collectivity (mesoscale organization of physical behavior) and flexibility (structural adjustment to guests).

Regarding the future of porous crystallites, Prof. Kitagawa categorized the importance of defects (or disorder), anisotropy and topological hierarchy. He illustrated these considerations using seminal works published over the last decade. The local vs. global dynamics of soft crystals and their interaction with guests was illustrated through molecular animations: CPL-7 distinguishing between $\mathrm{MeOH}$ and $\mathrm{CH}_{4}$, gate opening of interdigitated structures and the 
famous breathing behavior of Prof. G. Férey's MIL-53. We may be just at the beginning of the age of guest-responsiveness in porous materials.

A wide scope of looming applications was also highlighted. MOF-guest complexes as anhydrous proton transport membranes (engagingly illustrated by a movie of a spinning toy car connected to a fuel cell). $\mathrm{CO}_{2}$ capture from humid gas stream withholding capacity for 600 000 cycles (i.e. 6 years of projected use). Adsorption in MOFs as a low energy distillation alternative for some of the most challenging gas separations (Acetylene/ $\mathrm{CO}_{2}$ ). In vivo vasodilation studies using spatiotemporally controlled nitric oxide release by MOF nanoparticles. The ever-expanding horizon of potential applications for MOFs and related materials foreshadows the bright future of these materials. Moreover, further progress will be catalyzed by an ever improving set of characterization tools; femtosecond temporal resolution $\mathrm{X}$-ray diffraction using a free electron laser being a compelling recent example.

We can only conclude, in full agreement with Prof. Kitagawa, that whereas the science of porous crystals is clearly flourishing, the progress that is yet to be achieved by present-day junior researchers will probably go far beyond our current imagination. 